\title{
Una aproximación al análisis del balance entre oferta y demanda en las cercanías ferroviarias de la Comunidad de Madrid *
}

\author{
Gloria Fernández-Mayoralas Fernández ${ }^{\star \star}$
}

\section{INTRODUCCION}

El objetivo global del estudio se dirige a determinar el balance entre oferta y demanda ferroviarias, evaluando en qué medida, actualmente, existe correspondencia entre los niveles alcanzados por cada una de estas variables y qué mecanismos, temporales y espaciales, han contribuido, positiva o negativamente, en el proceso de su adecuación.

En este sentido, el planteamiento general de la investigación, se estructura en dos grandes bloques en función de estos componentes:

Por una parte, el configurado por la Oferta de Transporte. Esta ha sido considerada como un vector con tres factores (ROA, 1977):

- Primeramente, el de Dirección, que supone su actuación sobre una ruta o itinerario que, en el caso de la ferroviaria, se plasma físicamente en una infraestructura que le confiere una identidad particular y diferencial a otros

- Este artículo es una breve síntesis de la Tesis Doctoral “El transporte de viajeros por ferrocarril en la Comunidad Autónoma de Madrid", leida en la Facultad de Geografía e Historia en julio de 1988.

** IEGA-CSIC. 
modos terrestres. De un lado, porque depende de la propia empresa de transporte, siendo su uso exclusivo al ferrocarril, lo que condiciona favorablemente su capacidad circulatoria al impedir su agotamiento por congestión debida a otros medios. $Y$, por otro lado, porque sus elevados costes de implantación y, por tanto, de extensión, ofrecen una mayor rigidez a los cambios de la demanda.

- El segundo factor viene definido por el Sentido de Circulación, que complementa a la Dirección puesto que supone la existencia de relaciones bidireccionales o, lo que es lo mismo, un desglose de cada itinerario entre su ida y su vuelta.

- Por último, el tercer factor, la Magnitud, viene definido, de un lado, por la Capacidad Real de la Infraestructura, medida tanto en número de circulaciones como de plazas.

De otro lado, por la Distancia entre Origen y Destino.

Y, por fin, por la Calidad de los Servicios, que nosotros hemos expresado en términos de frecuencia, precio y tiempo invertido.

El otro gran componente del Sistema de Transporte, es la Demanda, que será planteada en función de las dos ópticas comúnmente utilizadas y no excluyentes entre sí:

- aquella que la concibe como derivada directamente de la necesidad de moverse (REICHMAN, 1983) y como resultado de una serie de factores extrinsecos $O$ independientes, relativamente, de la oferta;

- aquella otra que hace referencia a la posibilidad u oportunidad, real y concreta, de realizar la actividad de desplazarse, actuando, en la práctica, como un balance de uso o solicitando de la oferta, puesto que depende de las características de los servicios establecidos, tanto en Dirección, como en Sentido y Magnitud (ROA, 1977). 


\section{LA OFERTA DE TRANSPORTE FERROVIARIO}

La Infraestructura Ferroviaria que, como se ha dicho, es la plasmación física de la Dirección de la Oferta, se resuelve en la Comunidad en una red que presenta una estructura básicamente lineal, radial y centralizada en Madrid (Fig. 1).

Esta red es el resultado de la actuación de tres pautas u objetivos.

a) Destinado a conseguir un incremento de la Accesibilidad Global del Territorio Nacional mediante líneas que enlazaran la capital del Estado con los principales puertos y fronteras.

A este principio respondieron los tendidos de ancho normal durante el siglo pasado, primeramente hacia Aranjuez (1851) y Toledo (1858) y, más tarde, hacia Guadalajara (1859), Avila (1861-1863), Torrijos (1876), Ciudad Real (1979 y Segovia (1888).

b) La segunda pauta en la construcción, intentaba lograr un incremento de la cohesión o Accesibilidad Relativa del interior, también, del territorio nacional.

A este objetivo respondió la planificación del directo a Burgos en 1926, si bien, su apertura no se producirá hasta finales de los sesenta de este siglo.

c) Por su parte, la tercera pauta en los tendidos se basó en conseguir un incremento, también, de la Accesibilidad Relativa, pero, en este caso, del interior del territorio concreto de la Comunidad de Madrid.

Bajo este objetivo se encuadran los tendidos recientes a Cantoblanco (1975) y a Móstoles (1980-1983), cuyo desarrollo se vincula, si bien con cierto desfase, al crecimiento desmesurado de la metrópoli madrileña.

Anteriores a estas líneas de infraestructuras exclusivas al servicio de cercanías, sólo las de ancho métrico habian respondido a la necesidad de relacionar Madrid con su entorno próximo. Sin embargo, la mayor parte de ellas, con un tráfico prioritario de mercancias y explotadas por compañias de 
escaso nivel financiero, fueron levantadas paulatinamente a tenor de la competencia a que se vieron sometidas por el transporte por carretera.

Conforme a todo ello, el Análisis Topológico de la red traduce una escasa coherencia, con el desarrollo de un solo circuito y donde un subgrafo (el configurado por la línea de Laguna a Móstoles) permanece aún aislado (Fig. 1). Por otro lado, la representación gráfica de los índices de Shimbel revela un desplazamiento de la Accesibilidad Topológica hacia el Nor-Noroeste (Fig. 2), reflejo de que la mayor parte de los enlaces o estaciones se localiza en estos sectores, presentando, además, como nodo más accesible, topológicamente hablando, a la estación de Charmantín, a pesar de que es la de Atocha el centro real de las cercanías metropolitanas.

Puesto que esta red sólo relaciona 51 municipios de la provincia requiere de medios de transporte afluentes que permitan completar su cobertura espacial a los usuarios.

En la práctica, la afluencia de otros medios, de carácter colectivo, es mínima y, de hecho, los servicios interurbanos de viajeros por carretera mantienen una elevada competencia con la red ferroviaria, repitiendo las características de centralidad y radialidad, aunque ofreciendo un grafo más completo y compacto.

Por su parte, la Magnitud de la Oferta ha seguido unas pautas similares en su evolución a la del tendido infraestructural, donde se han solapado los tráficos de largo y corto recorrido, demostrándose un interés muy reciente sobre estos últimos y, especialmente, sobre las zonas espaciales demográficamente más dinámicas.

Ello se comprueba con el análisis de cada una de las tres variables que configuran este factor: la Distancia entre origen y destino, la Capacidad Circulatoria y la Calidad de los Servicios:

A) Por lo que refiere a la primera de ellas, asistimos, desde 1965 a un proceso que podriamos calificar como de "relleno" mediante el desarrollo de circulaciones entre Madrid y aquellas estaciones comprendidas en un radio de 30 a 50 kilómetros; distancia que define los recorridos de cercanias.

Este proceso se observa, primeramente, sobre los intinerarios del Noroeste (hasta Villalba) y Noreste (hasta Alcalá de Henares) y no se verificará en los del Sur y Soroeste (hasta Parla, Fuenlabrada y Móstoles) hasta 1981. 
B) Por lo que respecta a la Capacidad Circulatoria, y limitándonos a la cobertura municipal efectuada por cada itinerario, puede observarse (Fig. 3) cómo, el nivel exigido de un mínimo de 96 expediciones completas diarias para que un servicio sea considerado de cercanías, sólo será alcanzado a partir de 1975 por los términos municipales del Corredor del Henares (San Fernando, Torrejón y Alcalá), junto con Las Rozas y Collado-Villalba en el Noroeste, no llegándose a perfilar un predominio circulatorio sobre los sectores más poblados del Sur y Suroeste (Alcorcón, Móstoles, Leganés, Fuenlabrada, Getafe y Parla) hasta 1981.

Esta capacidad circulatoria municipal fue sometida a un Análisis de Regresión $n^{1}$ para medir la fuerza de asociación con ciertas variables que se estimaron a priori como condicionantes de la misma:

1. En primer lugar:

- La Población, tanto en cifras absolutas, como relativas expresadas en densidad por superficie.

- Su Estructura por Edades, expresadas en tasa de población joven y tasa de población adulta, como grupos demográficos que experimentan mayor movilidad.

- Su Estructura Ocupacional, medida a través de la tasa de ocupación en la industria y en los servicios, sectores de actividad que generan mayor número de desplazamientos.

- Yel Crecimiento Demográfico entre 1970 y 1981, para determinar el grado de dinamismo de la población.

2. En segundo lugar la Distancia a Madrid de cada uno de los términos municipales, que actúa como variable condicionante del grado de influencia de la capital como generadora de viajes cotidianos.

3. En tercer lugar, una serie de variables que hacen referencia a las Caracteristicas del Empleo Municipa/ determinadas a partir del número total

1 Se utilizó el Análisis de Regresión Múltiple por pasos del Programa BMDP2R. 
de establecimientos industriales, de los trabajadores empleados en industrias con más de 500 obreros, de la cantidad de establecimientos comerciales y de la superficie agraria.

4. Y, finalmente, dos variables referidas al Conjunto de Viviendas Construidas y, en concreto, a las de Uso Secundario, para determinar el grado de participación de otros factores de movilidad, como el ocio, sobre la magnitud de la oferta.

Un resultado para el Coeficiente de Correlación de 0,85 y para el de Derterminación de 0,72 , comprueba estadísticamente la existencia de una fuerte asociación, de signo positivo, entre la cobertura ferroviaria municipal y una sola de las variables testadas, la densidad de población.

El Análisis de los Residuos derivados de la contrastación entre los valores observados y esperados traduce una elevada asociación espacial entre ambas variables (Fig. 4) pues, la mayor parte de los casos presentan resultados próximos a cero.

Las desviaciones negativas, esto es, los términos municipales que presentan un déficit en su oferta conforme a los valores esperados, se localizan fundamentalmente en el Norte de la Comunidad. Se trata de una zona que, a pesar de registrar bajas densidades demográficas observa, también, una escasa magnitud circulatoria. Pero además, se advierten también residuales negativos en el Sur provincial, sobre términos municipales que, como Leganés y Parla, mantienen considerables concentraciones de población.

Por el contrario, los casos que poseen una oferta ferroviaria superior a la esperada conforme a su densidad demográfica, se localizan en el Noroeste (Las Rozas, Galapagar, Cercedilla y Santa María de la Alameda), y en los extremos provinciales de los itinerarios del Noreste (en Meco) y Sur (en Aranjuez), lo que, además de por coincidir con las relaciones ferroviarias tradicionalmente más favorecidas, se explica por la rigidez de este medio, que mantiene niveles similares de servicio a distancias superiores a lo que, según una demanda potencial de viajes cotidianos, cabría esperar.

Esta variable de Distancia sí que es recogida, sin embargo, como correlada cuando el análisis de regresión se realiza sobre el conjunto del transporte colectivo (es decir, considerando ambas ofertas, tanto por ferrocarril como por carretera), ya que los servicios de líneas regulares prestan 
una cobertura más flexible 0 , lo que es 10 mismo, con mayor sensibilidad a los cambios de demanda.

C) Por último, también la Calidad de la Oferta, establecida en términos de frecuencia, precio y tiempo de transporte, traduce, en su evolución, el reciente interés demostrado por los tráficos de corto recorrido o cercanías:

- La Frecuencia Circulatoria, no observarán pautas de homogeneización en la distribución de la capacidad total hasta 1981, y sobre aquellos servicios que mantienen mayor cantidad de expediciones: en los de Móstoles, Guadalajara y Villalba. Sin embargo, los del Sur, a Aranjuez, Toledo y Grinón, y, sobre todo, el de Cantoblanco, registran fuertes oscilaciones en la relación circulaciones/hora que soportan. Mientras que el directo a Burgos, mantiene una frecuencia mínima de dos expediciones completas en el conjunto de la jornada lo que, en todo caso, invalida su consideración como servicio de cercanías.

- El Precio del Transporte, por su parte, ha sufrido una paulatina desvalorización derivada del proceso inflacionista (Fig. 5), y lo que podria parecer un favorecimiento al usuario en realidad no ha hecho más que contribuir a una progresiva descapitalización del medio.

Sin embargo, a partir de 1981 se aprecia una relativa recuperación en los niveles. De hecho, entre 1981 y 1984 se registran los mayores incrementos en las tarifas lo que, no obstante, ha venido acompañado de la extensión de reducciones especiales sobre los precios específicos a las cercanías, favoreciendo la movilidad laboral que se desarrolla sobre ciertos sectores espaciales, en concreto, los servidos por las relaciones a Móstoles, Guadalajara, Aranjuez, Parla y Fuenlabrada, dando prioridad a los despalzamientos de carácter regular mediante los billetes de ida y vuelta o la implantación de abonos semanales, mensuales, etcétera.

- Por último, el tiempo de transporte, que actúa como un coste adicional al usuario, observa, también, la mayor reducción en la corona de cercanias, con respecto al resto, desde 1981. Sin embargo, aún el 30 por 100 de los usuarios sufre un incremento de más de un tercio en su tiempo de desplazamiento debido al 
que consume en el acceso y dispersión a la red, sin contar con que, además, más del 50 por 100 de los mismos necesita realizar al menos un cambio modal para alcanzar su destino.

Es en concreto sobre estos tiempos adicionales sobre los que una actuación destinada a su reducción puede servir como mejor catalizador de la demanda, ya que el tiempo propio de viaje es considerado, por la mayor parte de los usuarios, como un mal menor o, en todo caso, necesario.

\section{LA DEMANDA DE TRANSPORTE: CARACTERISTICAS Y FACTORES CONDICIONANTES}

Por su parte, el segundo gran componente del Sistema de Transporte, la Demanda, se define como el deseo, o el hecho en sí, de realizar la actividad de desplazarse y, como cualquier otra actividad, requiere de un Proceso de Decisión, con una serie de fases de las que se derivan sus características principales:

- En primer lugar, quién es el sujeto que decide (según su sexo, edad, nivel de renta, etc.), y, por extensión, cuál es el origen del desplazamiento, también conocido como Generación de Viajes (LANE, POWELL y PRESTWOOD, 1975).

- En segundo lugar, cuál es el motivo que mueve a la decisión (trabajo, estudios, ocio, etc.), con el que se relaciona el destino (también llamado Distribución de Viajes) que se elige en función de las prestaciones ofrecidas.

- En tercer lugar, la selección del modo de transporte o la ruta sobre la que se realizará el desplazamiento, que dependerá de las características de la oferta en cada medio.

De estos componentes iniciales se derivan, asimismo, otras variables de interés en los estudios sobre demanda: el momento elegido para realizar la actividad de desplazarse, que dependerá de la motivación del viaje pero también de las prestaciones de la oferta, y los costes que un individuo o 
grupo de individuos está dispuesto a soportar, y que actúan como resistencia o negatividades al deseo de viaje.

Estas variables pueden ser conocidas a través de las Encuestas OrigenDestino o Estudios de Opinión sobre Movilidad ${ }^{2}$.

En lo que concierne a Madrid, la mayor parte de estas Encuestas se limitan al área Metropolitana, lo que no es más que una consecuencia de que en torno al 90 por 100 de los desplazamientos se realizan en este sector espacial de la Comunidad. La heterogeneidad en los presupuestos de partida de cada uno de estos estudios (en la elección zonal, en los sujetos a los que se dirigen, etc.) restringe, en cierta medida su comparación, no obstante, en líneas generales se pueden apuntar las siguientes conclusiones:

- Primeramente, por lo que se refiere al sujeto no se observan diferencias de movilidad global en cuanto al sexo, aunque sí conforme al modo de transporte utilizado por cada uno. En este sentido, la mujer, por su escasa incorporación al trabajo, generalmente se desplaza a pie $y$, cuando precisa desplazarse a mayor distancia, se muestra más cautiva de los transportes públicos que el varón, sujeto productivo y el que normalmente detenta la propiedad del automóvil privado.

Por otro lado, el mayor número de los desplazamientos son efectuados por individuos con edades comprendidas entre los 25 y 34 años. Entre los i 8 y 24 se observa una mayor dependencia de los medios públicos, mientras que, relacionado también con la incorporación a actividades productivas, los medios privados son más utilizados por sujetos entre 25 y 34 años.

Finalmente, tampoco se observa una correlación perfecta entre el nivel de motorización y el uso de medios públicos pues si, de hecho, la ausencia de automóvil propio sí condiciona su uso, la propiedad de más de un vehículo, no significa, necesariamente, que no se vayan a utilizar. Lógicamente, la mayor parte de los usuarios de los medios colectivos no poseen vehículo propio, sin embargo, se establece, también, una importante participación de aquellos que sí cuentan con automóvil disponible, advirtiéndose una relación menos desfavorable al modo ferroviario con respecto al bus regular.

\footnotetext{
2 Encuesta O/D de: GALLUP (1974), EMOPUBLICA (1979) e INECO (1981).
} 
- En lo que respecta a los motivos que incitan a desplazarse aparecen como causas más explicativas de la demanda los viajes al trabajo y por estudios, generando aquéllos en torno al 50 por 100 de los desplazamientos. Se advierte, también, cómo ambos motivos se ajustan mejor a la radialidad del sistema ferroviario, como se demuestra al comparar su participación sobre el total de medios colectivos.

- En cualquier caso y para todos los motivos, los modos mecánicos más utilizados son los públicos, y, de ellos, el que más proporción muestra, es el autobús regular, pues no en vano es el que mantiene una cobertura espacial más amplia.

- En lo que concierne a la asignación de rutas, las demandas más acusadas sobre los medios colectivos se registran sobre los itinerarios que cubren el Sur, Suroeste, Noreste y Corredor del Henares.

Ciñéndonos a la movilidad suscitada sobre los servicios ferroviarios, puede observarse en la tabla I cómo, entre las relaciones a Móstoles y a Alcalá de Henares soportan casi el 57 por 100 de la demanda, lo que, junto con el resto de los servicios del Sur (a Aranjuez, Parla y Fuenlabrada) se eleva al 85 por 100 de la total desarrollada. Por otro lado, los desplazamientos de carácter laboral, observan una presencia mayor sobre los ejes de Alcalá, Parla, Fuenlabrada y Móstoles. Mostrando, tanto el motivo 'estudios' como los viajes No Basado en el Domicilio, la mayor proporción sobre la relación de Madrid a Cantoblanco.

- Finalmente, por lo que se refiere a la participación, según el grado de motorización de los demandantes sobre cada eje ferroviario resalta que, además de registrarse los mayores niveles sobre las relaciones del Noroeste, lo que resulta lógico dado el estatus socioeconómico de la población de este sector, el servicio a Móstoles, y, en menor medida, los de Parla y Fuenlabrada, presentan una solicitación relativamente elevada de usuarios con vehículo propio y disponible (tabla II). Ello confirma un mayor desarrollo de los índices de motorización en estas zonas, a pesar de rentas inferiores y como respuesta a ofertas inexistentes, escasas o de deficiente calidad, en los servicios ferroviarios del Sur que, hasta fechas recientes, no observaron importantes incrementos en la magnitud de su oferta, o del Suroeste, que también recientemente, ha conseguido contar con un ferrocarril especifico de cercanías. 


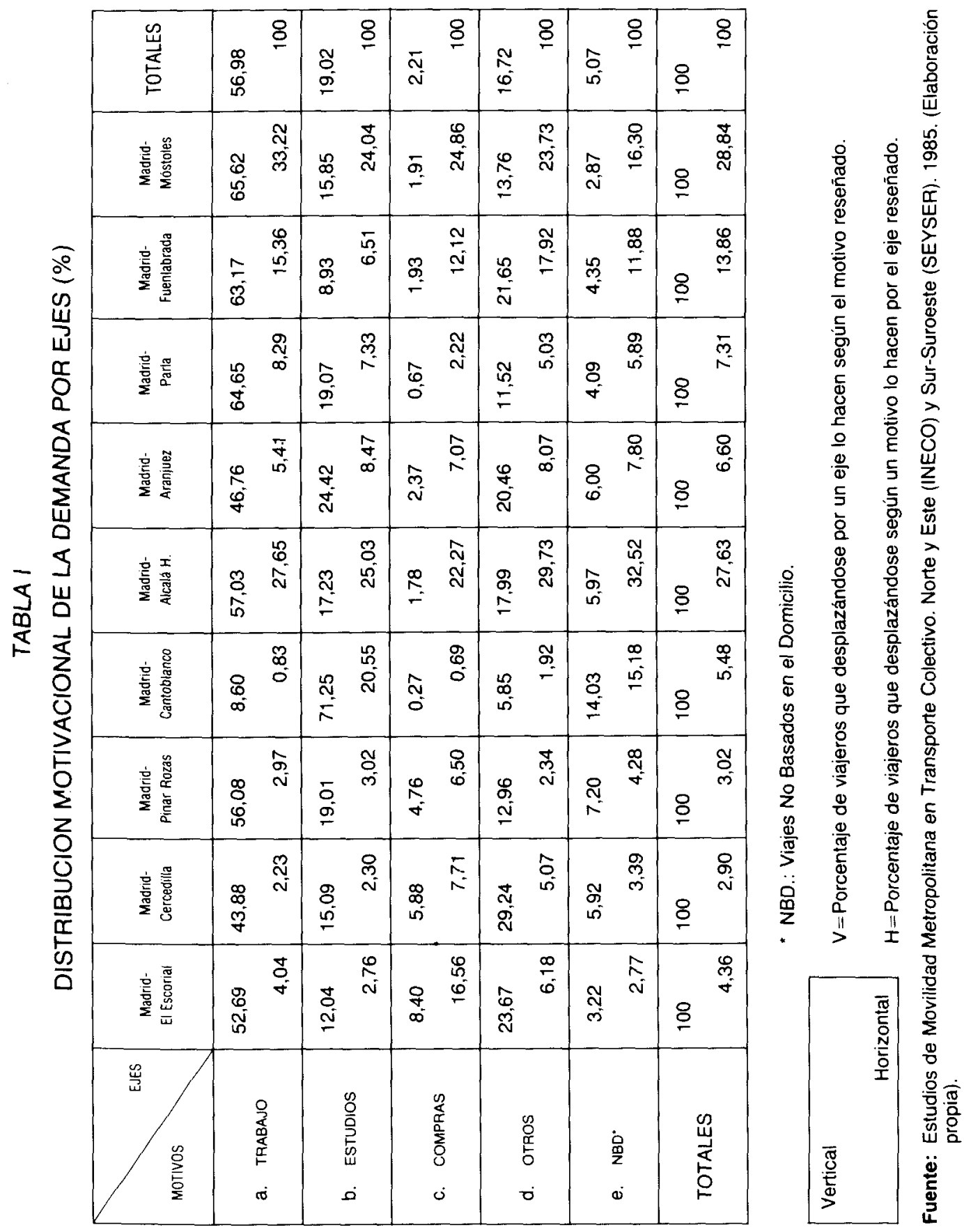




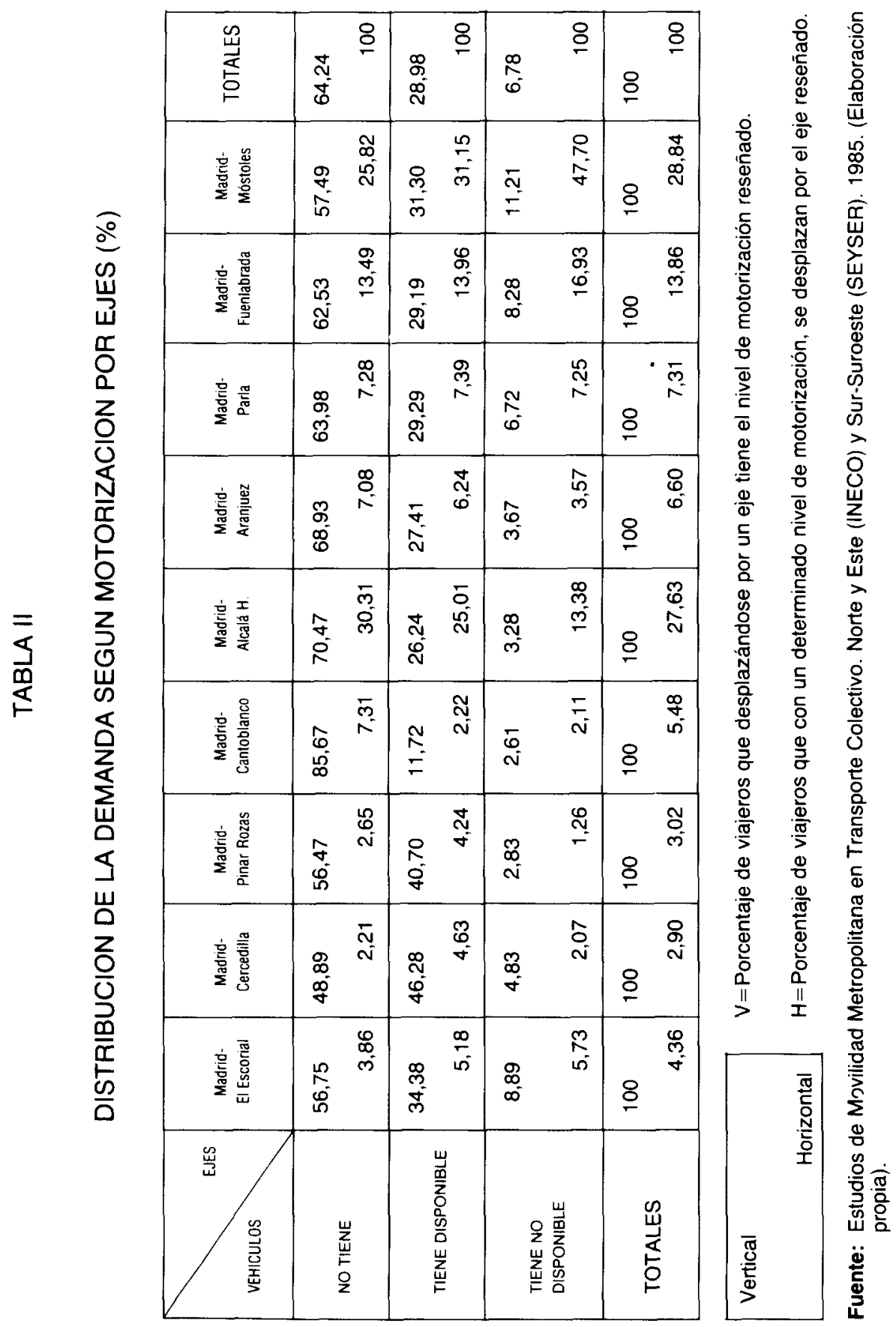


Esta demanda, que hemos expuesto brevemente en sus características principales, responde a una serie de factores (PEREZ SANZ y VAZQUEZ, 1982) que hacen referencia a los aspectos siguientes:

1) La localización espacial tanto de la población como del empleo y los equipamientos;

2) la distribución de otros componentes sociales generadores de movilidad;

3) la legislación y planificación de los usos y modos del transporte;

4) el propio sistema de transporte en cuanto a las características de su oferta, enlazando ya con la aproximación económica de la demanda que ve en ésta el resultado del aprovechamiento que se establece sobre los distintos servicios (ROA, 1977).

1. La población influye directamente sobre la magnitud de la demanda a través de su volumen total, pero también, y más importante, en razón a su localización espacial diferencial con respecto a la oferta de empleos y equipamientos en general.

Ya hemos visto cómo los servicios más demandados se corresponden con aquellos sectores más vitales demográficamente, y en función, sobre todo, a los viajes generados por motivo de trabajo.

En este sentido, un reciente estudio, realizado en 1980 para el CEOTMA, sobre la Movilidad entre Madrid y su Area Metropolitana ${ }^{3}$, confirma como zonas mejor dotadas de empleos, esto es, con saldos positivos, a la Capital, el Noroeste y el Corredor del Henares, y la existencia de saldos negativos en el resto del Area.

Sin embargo, el número de individuos que deben desplazarse fuera de su lugar de residencia a trabajar, supera, en todos los casos, el déficit observado de empleos, existiendo, incluso, generación de flujos de activos desde las zonas con saldos positivos.

${ }^{3}$ Encuesta sobre Movimientos Recurrentes y No Recurrentes entre Madrid y su Area Metropolitana (EMOPUBLICA). 
De estos flujos, casi el 94 por 100 se producen radialmente por el intercambio bidireccional entre Madrid y el resto de los conjuntos espaciales, dibujando, el 62 por 100 restante, flujos interzonales de menor entidad y de carácter transversal, sobre todo, entre las localidades comprendidas desde el Oeste hasta el Corredor, por el Sur del Area.

Ciñéndonos a los flujos radiales, puede apreciarse en la tabla III cómo las direcciones básicas se perfilan entre Madrid y los conjuntos espaciales del Sur (esto es, Getafe, Parla, Leganés y Fuenlabrada), Oeste (Alcorcón, Móstoles, Villaviciosa, Brunete y Villanueva de la Cañada) y Corredor del Henares (Coslada, San Fernando, Torrejón, Alcalá, Meco, Mejorada y Paracuellos).

El sentido de estos flujos se muestra más positivo a las entradas en la capital desde todas las zonas, a excepción del Corredor, Noroeste y Pinto. Recordemos que, si exceptuamos este último, de escasa magnitud, aquéllos se corresponden con los conjuntos mejor dotados de empleos, junto con el de Madrid.

Aparte de estos desequilibrios descritos entre población y empleo, otras variables (sobre las que no nos extenderemos en este momento), como la localización espacial de determinados equipamientos, actúan, también, como generadoras de movilidad.

2. El segundo de los Factores condicionantes de la Demanda de Transporte se refiere a la distribución de otros componentes sociales generadores de movilidad, entre los que se incluyen las variables de renta familiar disponible y nivel de motorización.

La interrelación entre ambas ha sido repetidamente constatada: un mayor nivel de renta posibilita la adquisición de uno o más vehículos.

Sin embargo, puede apuntarse que la asociación no siempre es perfecta, puesto que el acceso al primer vehículo no es independiente al desequilibrio descrito entre población y actividades, ni a la cobertura prestada por las distintas redes de transporte, por lo que a igualdad de renta pero mayor densidad de movilidad y menor oferta en transporte colectivo, el nivel de motorización habrá de incrementarse necesariamente.

3. El tercero de los Factores condicionantes de la Demanda hace referencia a los aspectos derivados de la política de transporte, en los que nosotros 
hemos advertido la causa última del desfase entre oferta y demanda ferroviarias, por cuanto que este modo se ha visto relegado de resultas del impulso prestado a los medios por carretera, tanto públicos como privados, por considerarlos más apropiados para lograr el modelo previsto de concentración territorial.

Efectivamente, durante el presente siglo, la historia del ferrocarril se ha caracterizado por el déficit financiero que, agravado por los desastres de la Guerra Civil, había provocado una importante descapitalización del medio, lo que, a su vez, vino a propiciar la incipiente competencia de los medios por carretera.

Las leyes de Ordenación y Coordinación de los Transportes Mecánicos Terrestres de finales de los 40 , no crearon un marco legislativo eficaz para el desarrollo coordinado real entre modos, pues, si en el papel se consideraba a la carretera como complementaria del ferrocarril, en la práctica, los supuestos contemplados como de interés público, que justificaban la extensión de los medios por carretera, se impusieron.

De hecho, en torno a las grandes ciudades y, en concreto, en la de Madrid, la prioridad concedida al transporte ferroviario quedaba abolida, puesto que los servicios regulares de viajeros por carretera que fueran coincidentes con el ferrocarril, serían considerados como independientes siempre que la longitud de sus itinerarios representara un acortamiento superior a) 25 por 100 o, también, cuando enlazaran núcleos urbanos importantes con aglomeraciones suburbanas, siempre que la longitud no excediera de 50 kilómetros si el núcleo principal no superaba el millón de habitantes.

Por otro lado, y enmarcada dentro de una política general de concentración de recursos en una serie de Areas Urbanas, la política inversora del Estado se volcó sobre la mejora y construcción de la Red de Carreteras, comenzándose a hablar de racionalizar la RENFE mediante el cierre de líneas y la potenciación únicamente de su Red Básica.

La crisis económica de los 70 vino a poner en entredicho, no tanto este modelo territorial, situación de desequilibrio que, como hemos visto, provoca intensos desplazamientos, sino el uso excesivo del automóvil privado, de bajo aprovechamiento, menor rendimiento energético y elevados costes sociales asociados, que genera, además, un rápido agotamiento de la capacidad vial, entorpeciento los servicios públicos de transporte por carretera. 
Se planteará, entonces, la necesidad de uso del transporte colectivo y, en concreto, del ferroviario, pues resulta el más eficaz para aliviar la congestión rodada en las áreas urbanizadas, desarrollándose la idea de $\mathrm{Cer}$ canías que, en Madrid, se plasmará en la paulatina intensificación de la oferta en los recorridos metropolitanos, en el tendido de nuevos viarios de índole suburbana y en la Creación de una Jefatura, ya en 1981, que gestionara la movilidad generada con este carácter.

A este cambio de concepción sobre el transporte por ferrocarril responde el Plan Ferroviario para el Area Metropolitana de Madrid ${ }^{4}$ que plantea la racionalización del espacio ferroviario a partir de la especialización de las infraestructuras según el tráfico que soporten (mercancias, viajeros-largo recorrido, 0 viajeros-corto recorrido) y su accesibilidad al uso principal, manteniendo el principio de integración del ferrocarril como un elemento más del mobiliario qie define el paisaje urbano.

En este sentido, el Plan viene a resaltar el papel de las Cercanías al considerarlas no subsidiarias del resto de los tráficos, así como la importancia de facilitar el acceso a las infraestructuras mediante el desarrollo de estaciones de intercambio modal que hagan físicamente posible la coordinación entre los diferentes modos de transporte.

El marco legislativo para esta coordinación en la Comunidad de Madrid es aún más tardío, pues no será hasta 1985 cuando se apruebe la Ley de Creación del Consorcio Regional de Transportes, organismo autónomo de la Comunidad que concentra las competencias sobre transporte público regular de viajeros, asi como de los Ayuntamientos que deseen adherirse, y cuya única relación con la RENFE se instrumenta mediante la firma de Contratos-Programa.

Todas estas medidas coordinadoras que básicamente pretenden conseguir un uso preferente del transporte ferroviario en las relaciones radiales que, como se ha visto, son las que mayor demanda soportan en el ámbito metropolitano, facilitando el acceso entre modos a través de intercambiadores, unificando tarifas y disuadiendo del uso del automóvil privado mediante la construcción de aparcamientos, coinciden sustancialmente con las que comenzaron a tomarse en diversos países industrializados desde los años 70, advirtiéndose un importante retraso en España, lo que ha provo-

${ }^{4}$ Elaborado a partir del Convenio firmado en 1982 entre el Ayuntamiento, el Ministerio de Transportes, la antigua COPLACO y la RENFE. 
cado un intenso desfase entre la oferta global de transporte colectivo con respecto a las necesidades de movilidad cotidiana generadas en torno a las grandes ciudades y, en concreto, a la de Madrid.

4. El cuarto y último factor que apuntamos como condicionante de la Demanda hace referencia a las caracteristicas de los servicios de transportes establecidos, en Dirección, Sentido y Magnitud, enlazando con la aproximación aconómica de los estudios sobre capacidad, que consideran la demanda resultado de la solicitación de la oferta, actuando, en la práctica, como un balance de uso o aprovechamiento.

Según su reciente estudio realizado por la Jefatura de Cercanías de la RENFE sobre los Resultados de Explotación de las Cercanías de Madrid, el número de viajeros transportados diariamente, en 1981, alcanzaban la cifra media de 110.000, registrándose un incremento de la demanda, entre esta fecha y 1983, del orden del 27 por 100, y fundamentalmente sobre las relaciones ferroviarias de Parla y Fuenlabrada.

Las direcciones que absorben una mayor proporción de viajeros se corresponden con los itinerarios a Móstoles y Guadalajara, que superaban el 65 por 100 en 1981, apreciándose un decremento al 58 por 100, en el final del periodo.

\section{LA DEMANDA DE USO: BALANCE OFERTA-DEMANDA}

Conocido el tamaño global de la demanda de uso, expondremos a continuación el proceso que seguimos para delimitar la actuación de cada una de las variables o factores dependientes de la oferta sobre el comportamiento distributivo de esta demanda.

Estos FACTORES son los siguientes:

A) Por un lado, los itinerarios (I).

Este factor cuenta con seis niveles, que se corresponden con las relaciones de:

- Madrid-Villalba-Avila. 
- Madrid-Villalba-Segovia.

- Madrid-Guadalajara.

- Madrid-Aranjuez.

- Madrid-Parla-Toledo, y

- Madrid-Fuenlabrada-Griñón.

Quedan excluidos pues, los servicios de:

- Laguna a Móstoles, pues no aparece recogido en la fuente consultada.

- El de Madrid a Riaza, porque los niveles de su oferta no lo indican como un servicio de cercanías.

- Los de Príncipe Pío a Pinar de las Rozas y Atocha a Cantoblanco, pues sus pequeñas longitudes los hacian susceptibles de análisis sin recurrir al muestreo de la información.

B) El segundo factor hace referencia al sentido de circulación (S) de los trenes. Este factor cuenta con dos niveles:

- El determinado por las salidas de expediciones de Madrid capital.

- Y el definido por las llegadas a Madrid.

C) El tercer factor hace referencia al horario del tren $(\mathrm{H})$, con 20 niveles horarios de 60 minutos cada uno, establecidos entre las 5.00 de la mañana, hasta la 1.00 a.m. del día siguiente.

D) El cuarto factor se refiere a los tramos de itinerario o coronas (C), que presenta seis niveles para cuya delimitación se compaginaron criterios de homogeneidad espacial, en el interior de cada tramo, junto con la cate- 
goria de circulación entre las estaciones que se tomaban como límites de cada corona.

E) El quinto factor viene definido por los meses de circulación (M), estableciéndose los 12 niveles naturales.

F) Por último, el sexto factor es el de los años, con dos niveles, el de 1981 y el de 1984.

La fuente que se consultó, los listados mensuales sobre aprovechamiento de plazas en trenes de cercanias ${ }^{5}$, contienen información sobre viajeros desplazados y plazas ofrecidas, por kilómetro, en cada tren, por su tipo (ómnibus, ferrobús o unidades eléctricas), su horario de salida y llegada y el número de días de circulación, en el mes referido. No identifica, en cambio, los días de circulación con lo que los trenes no pueden ser tipificados semanalmente.

Sobre esta información se efectuó un sondeo por la Técnica de Muestreo Aleatorio Simple, con el objetivo de determinar si los seis factores considerados influían sobre el comportamiento de la demanda con la misma intensidad, y/o si el número de niveles podia ser reducido por agrupamiento en alguno de los factores.

Las observaciones se realizaron para cada tren seleccionado sobre las variables 'viajeros' y 'plazas'.

De este primer sondeo se extrajeron dos tipos de Conclusiones:

A) En lo relativo al número de plazas, se observó que no mostraban variación significativa entre los distintos factores, pudiendo ser considerado como una constante, salvo las excepciones que se detallarán más adelante. En este sentido, los sondeos siguientes no incluyeron este dato.

B) En lo relativo al número de viajeros, se observó una gran variabilidad en sus distribuciones con respecto a los factores referidos a ltinerarios, Sentido

\footnotetext{
${ }^{5}$ Elaborados por el Gabinete de Informática de la RENFE.
} 
de Circulación y Años Sondeados, lo que permitió su 'fijación', es decir, extraerlos del Modelo Hexafactorial inicial, y sobre ellos estudiar la distribución de los tres factores restantes: los Horarios, las Coronas y los Meses.

Una segunda serie de sondeos mostró que era posible, igualmente, una reducción de los niveles en los factores no fijados. Así, el factor Meses, que no registraba variaciones significativas entre los 12 niveles planteados, pudo reducirse a seis niveles bimensuales sin pérdida de generalidad. $Y$, por adecuación a los factores Coronas y Meses, los 20 niveles del factor Horarios fueron también reducidos a seis (de tres horas cada uno y con dos intervalos abiertos, el primero antes de las 9.00, y el último, después de las 21.00), lo que resulta un ajuste de clases con las utilizadas en los Estudios de Movilidad Metropolitana ${ }^{6}$.

Según todo ello, la hipótesis de partida, que establecía que una observación era debida a los efectos individuales y combinados de los seis factores descritos, quedó simplificada a 24 Modelos Trifactoriales (24 es el resultado de la combinación de los tres factores fijados con sus niveles: seis de los Itinerarios, por dos que corresponden con los Sentidos de Circulación y por dos que se corresponden con los Años planteados), expresados por la notación siguiente:

$$
Y i j k=\mu+C i+H j+M k+(C H) i j+(H M) j k+(C M) i k+(C H M) i j k+\varepsilon i j k
$$

donde,

Yijk es la observación ijk-ésima, o el valor de la observación cuando el factor Coronas tiene el nivel $i$, el factor Horarios toma el nivel $j$ y el factor Meses tiene el nivel $k$.

$\mu \quad$ es la media general de las observaciones.

$\mathrm{Ci}, \mathrm{Hj}$ y $\mathrm{Mk} \quad$ son los efectos individuales debidos a los niveles $i, j$ y $k$ en los factores Coronas, Horarios y Meses.

${ }^{6}$ Realizados para la Comunidad de Madrid por las Consultoras INECO y EYSER, en 1984. 
El resto de los términos hace referencia al conjunto total de las combinaciones bifactoriales y trifactorial de los tres factores, y, por último, $\varepsilon$ ijk es el efecto debido al azar.

Con este Modelo General Trifactorial Completo, se contrastó un tercer sondeo de observaciones para cada una de las 24 combinaciones ItinerarioSentido de Circulación-Año (ISA).

Su contrastación demostró que el factor Meses no presentaba un peso específico en las diferencias muestrales observadas, es decir, la demanda, sobre las cercanías ferroviarias de Madrid, no se encuentra sometida a oscilaciones de carácter estacional, propias de otro tipo de servicios, ya sean mercancías o viajeros de largo recorrido. Como excepción, aparece el itinerario de Madrid a la Universidad de Cantoblanco, cuya demanda se concentra claramente sobre las expediciones que se realizan con carácter lectivo, lo que es resultado de la especificidad de su cobertura.

El resto de las relaciones contrastadas con el Modelo, mostraron ajuste a una de las cuatro pautas de comportamiento siguientes:

$$
\text { 1. } Y i j=\mu+C i+H j+\varepsilon i j
$$

Se trata de un Modelo Bifactorial Incompleto, donde el peso condicionante de la observación viene dado por el efecto independiente de los factores Coronas y Horarios.

A este modelo se ajustan 14 de las 24 Combinaciones Itinerario-Sentido-Año, lo que viene a identificar la tónica general del comportamiento de la demanda en las cercanias de Madrid.

Es interesante destacar que, en 1981, sólo se ajustaban los itinerarios del Noroeste, Corredor y Sur (a Aranjuez) que, como se recordará, son los tradicionalmente más favorecidos en su oferta, adatándose, más pronto, a una cobertura metropolitana. De hecho, en 1984, todos los itinerarios, en su sentido de salida de Madrid, muestran un ajuste de su demanda a esta pauta.

2. $Y i j=\mu+C i+H j+(C H) i j+\varepsilon i j$ 
Es también un Modelo Bifactorial, en este caso, Completo, pues la observación depende de cada uno de los factores (Coronas y Horarios) además del producido por efecto conjunto de ambos.

A este Modelo se ajustan seis Combinaciones ISA, que se corresponden, fundamentalmente, con los itinerarios que, en 1981, cubrian las zonas más dinámicas del Sur, el de Madrid a Parla y el de Madrid a Fuenlabrada, y que no registraban aún niveles propios a una oferta de cercanias.

3. $Y i=\mu+C i+\varepsilon i$

Esta pauta se corresponde con un Modelo Unifactorial donde las diferencias estadísticas en las observaciones se explican por el efecto individual del factor Coronas.

A este modelo sólo se ajustan tres Combinaciones Itinerario-SentidoAño, que se corresponden con los itinerarios de Madrid a Avila y Madrid a Guadalajara, en su sentido de vuelta o entrada en la capital, que no observan variaciones importantes en su demanda con respecto al horario de las expediciones, lo que constata una importante regularidad en la movilidad suscitada sobre estas circulaciones.

4. $Y j=\mu+H j+\varepsilon j$

Este cuarto Modelo es también de carácter Unifactorial, donde el efecto condicionante de la observación proviene, en este caso, de los Horarios de los trenes.

A esta pauta sólo se ajusta una Combinación ISA, la de Madrid a Avila en su sentido de vuelta y en 1981 , que no registra variaciones de demanda con respecto a la distancia a la capital.

\section{CONCLUSIONES}

De todo lo expuesto se deduce, en primer lugar, una serie de conclusiones generales, de carácter cualitativo, sobre los factores que condicionan 
las variaciones de la demanda sobre los servicios ferroviarios de cercanías de Madrid:

a) De un lado, ya se comentó, la demanda no se ve sometida a oscilaciones estacionales significativas.

b) Por otro lado, las oscilaciones semanales, que como se recordará no pusieron ser recogidas como factor pues la fuente no especifica el día concreto de circulación, no producen, sin embargo, ninguna distorsión en el Modelo, esto es, no se registran diferencias significativas de demanda atribuibles a que la expedición se realice en laborable o en festivo.

c) Sí se observan, en cambio, oscilaciones significativas en la demanda conforme a la distancia a Madrid (medida por el factor Coronas) o en razón al horario de circulación de los trenes.

Pero además de las conclusiones cualitativas, y a partir del análisis de las magnitudes que asumen las oscilaciones descritas en función del efecto que produce el factor horarios, pueden clasificarse los itinerarios en los siguientes grupos:

A) Aquellos que presentan un ajuste fundamental de su ocupación a los horarios laborales en función de una mayor oferta de empleos en la capital, con un aprovechamiento máximo sobre las circulaciones que hacen su entrada en Madrid entre las 5.00 y las 9.00 de la mañana, y su salida entre las 18.00 y las 21.00 .

Con este grupo se corresponden los servicios que cubren las zonas más deficitarias en empleos: los itinerarios de Madrid a Móstoles, a Fuenlabrada y a Parla.

B) El segundo grupo comprende los itinerarios que cubren sectores donde la relación población-empleo resulta elevada y, por tanto, los intercambios laborales se efectúan en ambos sentidos de circulación, superponiéndose horariamente entre sí y con los viajes motivados por otras causas.

A este grupo pertenecen los itinerarios del Noroeste, a El Escorial y a Cercedilla, con ocupaciones superiores sobre las expediciones que hacen 


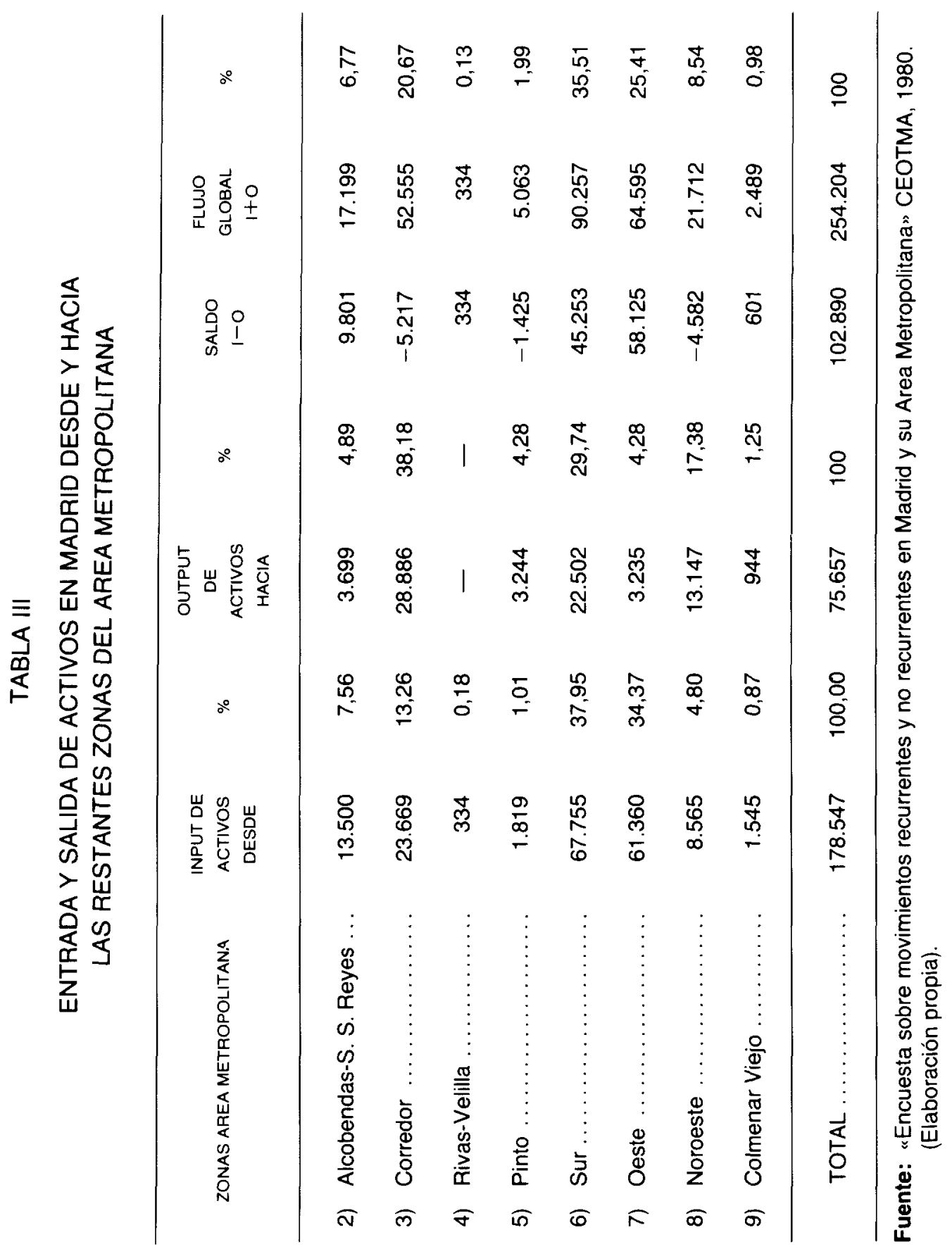


su entrada en la capital entre las 9.00 y las 12.00 y las 18.00 a las $21.00, y$ que tienen su salida de Madrid también en este último periodo horario.

Por su menor longitud y, por extensión, del tiempo invertido en el viaje, se incluye, igualmente, la relación de Príncipe Pio a Pinar de las Rozas, que no experimenta, en cambio, diferencias significativamente importantes en los niveles de ocupación que su demanda mantiene sobre las circulaciones que ofrece.

C) El tercer grupo comprende el servicio que cubre el Corredor del Henares que presenta oscilaciones importantes de aprovechamiento sobre las expediciones de salida de Madrid, con unos máximos de ocupación que se extienden entre las 5.00 y las 15.00 , y con apenas variaciones horarias, estadísticamente significativas, sobre las circulaciones con destino a la capital. Todo ello, acorde con la tónica de distorsiones que produce Guadalajara en la influencia de Madrid sobre su entorno provincial.

D) Por fin, el último grupo viene definido por la relación de Madrid a Cantoblanco, con ocupaciones de la oferta condicionadas por los horarios lectivos de la Universidad: mayores sobre las expediciones con salida de la capital en los dos primeros periodos horarios (de 5.00 a 9.00 y de 9.00 a 12.00) y en el cuarto (de 15.00 a 18.00), y sobre las de entrada en Madrid en el segundo, tercero y quinto (esto es, de 9.00 a 12.00, de 12.00 a 15.00 $y$ de 18.00 a 21.00).

Pero, aparte de las variaciones temporales del aprovechamiento, y en segundo lugar, son de resaltar también las oscilaciones espaciales observadas según la longitud del trayecto que se ve sometida a concentraciones significativas en la ocupación, distinguiéndose, entonces, tres tipos de itinerarios conforme a la distancia que alcanza la movilidad suscitada por los intercambios con la capital:

a) Primeramente, el Noroeste, con las relaciones a Avila y Segovia, que registran un aprovechamiento similar de la oferta hasta la cuarta corona que incluye a Villalba.

b) En segundo lugar, el Sur y Suroeste, con ocupaciones importantes hasta la tercera corona que comprende Valdemoro, Parla y Fuenlabrada; en este grupo se encuentran incluidos también los tendidos de menor longitud a Móstoles, Cantoblanco y Pinar de las Rozas. 
c) En tercer y último lugar aparece el itinerario del Noreste que presenta, una distorsión espacial en la distribución de la ocupación de la oferta sometida a niveles superiores de demanda, sobre las coronas más distantes de Madrid y en función de la influencia que ejerce Guadalajara sobre las localidades del Corredor.

Finalmente y por lo que se refiere a la relación entre oferta y demanda, medida ahora aquélla a partir del número de plazas ofrecidas por cada circulación, es interesante constatar que, del conjunto de los itinerarios, sólo tres efectúan alteraciones significativas sobre la capacidad tipo que había resultado fijada en 260 plazas-tren.

Estos tres itinerarios son el de Fuenlabrada, que junto con el de Cantoblanco refuerzan su oferta superando las 300 plazas, y el de Príncipe Pío a Pinar de las Rozas que, por el contrario, las reduce a una cifra inferior a 200 plazas.

Comparando ambas distribuciones, la de viajeros-tren y la de plazastren, hemos apreciado que los desajustes mayores se producen en las relaciones de Madrid a Parla, Madrid a Fuenlabrada y Madrid a Móstoles, fundamentalmente sobre las circulaciones que se ven sometidas a importantes concentraciones de demanda, es decir, aquellas que hacen su entrada en la capital antes de las 9.00 de la mañana y que, por tanto, se derivan claramente de la movilidad suscitada por los desequilibrios descritos entre población y empleo, que se muestran más acentuados, como hemos visto, en las localidades situadas en el Sur y Suroeste de Madrid, que, al mismo tiempo, son las que más recientemente han comenzado a ver su oferta ferroviaria adaptarse a una cobertura de cercanías para desplazamientos metropolitanos. 


\section{BIBLIOGRAFIA}

Lane, R.; Powell, T. y Prestwood Smith, P. (1975): Planificación Analítica del Transporte. IEAL Madrid, 351 pp.

Pérez Sanz, J. J. y Vazquez, G. (1982): Estudio del Sistema de Transportes en el Area Metropolitana de Madrid. Diputación de Madrid. Madrid, $96 \mathrm{pp}$.

Reichman, S. (1983): Les Transports: Servitude ou Liberté? PUF. París, $197 \mathrm{pp}$.

ROA, C. (1977): "Conceptos básicos sobre el estudio integral del Transporte». En III Curso de Planificación Territorial, 75. Centro de Perfeccionamiento Profesional y Empresarial. Madrid, páginas 149-177. 


\section{FIGURA 1}

GRAFO DE LA RED FERROVIARIA-1984

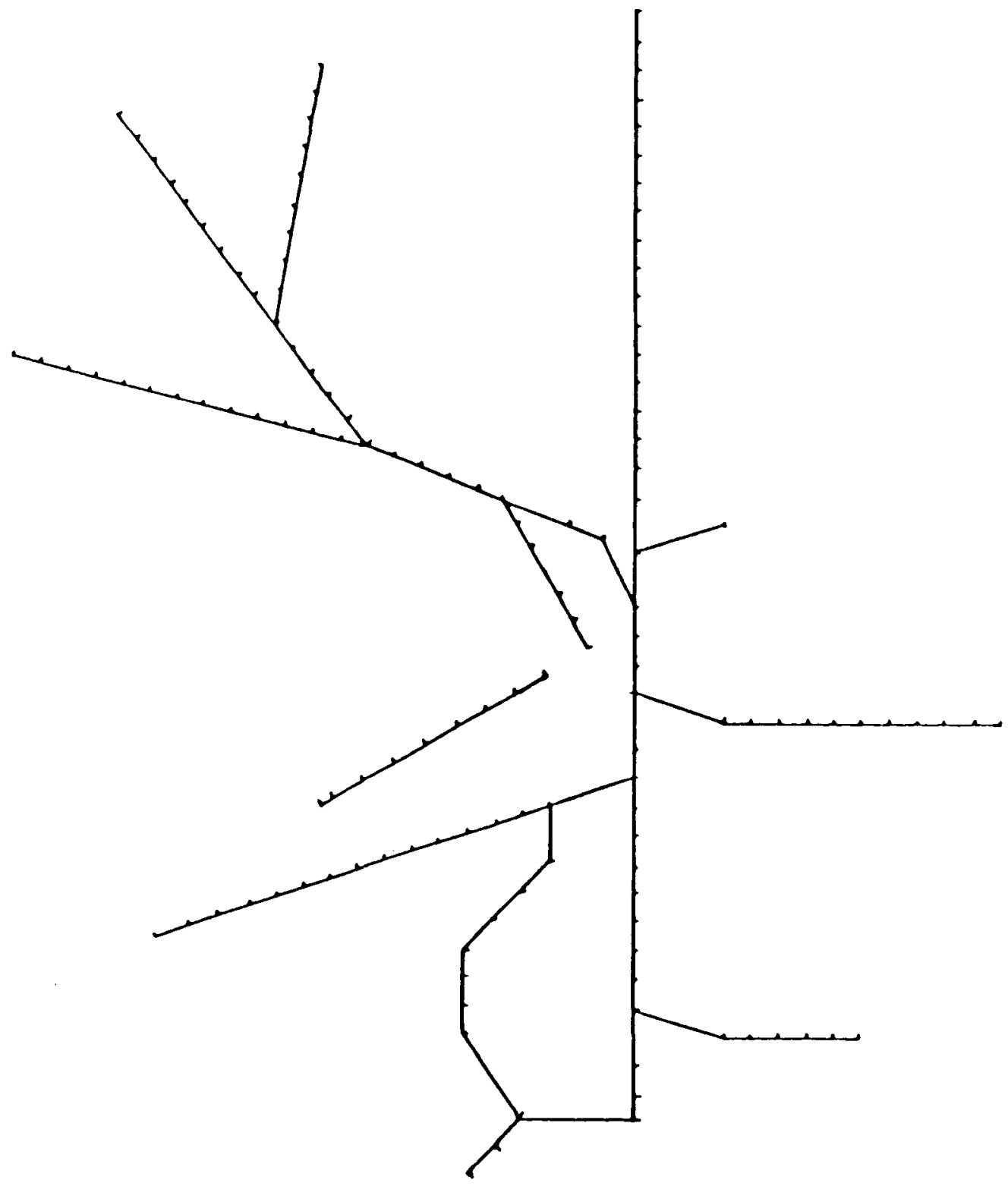

Fuente: Guias Comerciales de RENFE. (Elaboración propia). 
Una aproximación al análisis del balance entre oferta y demanda

\section{FIGURA 2}

ISOLINEAS DE ACCESIBILIDAD TOPOLOGICA ENTRE LOS NODOS DE LA RED FERROVIARIA TOTAL 1984

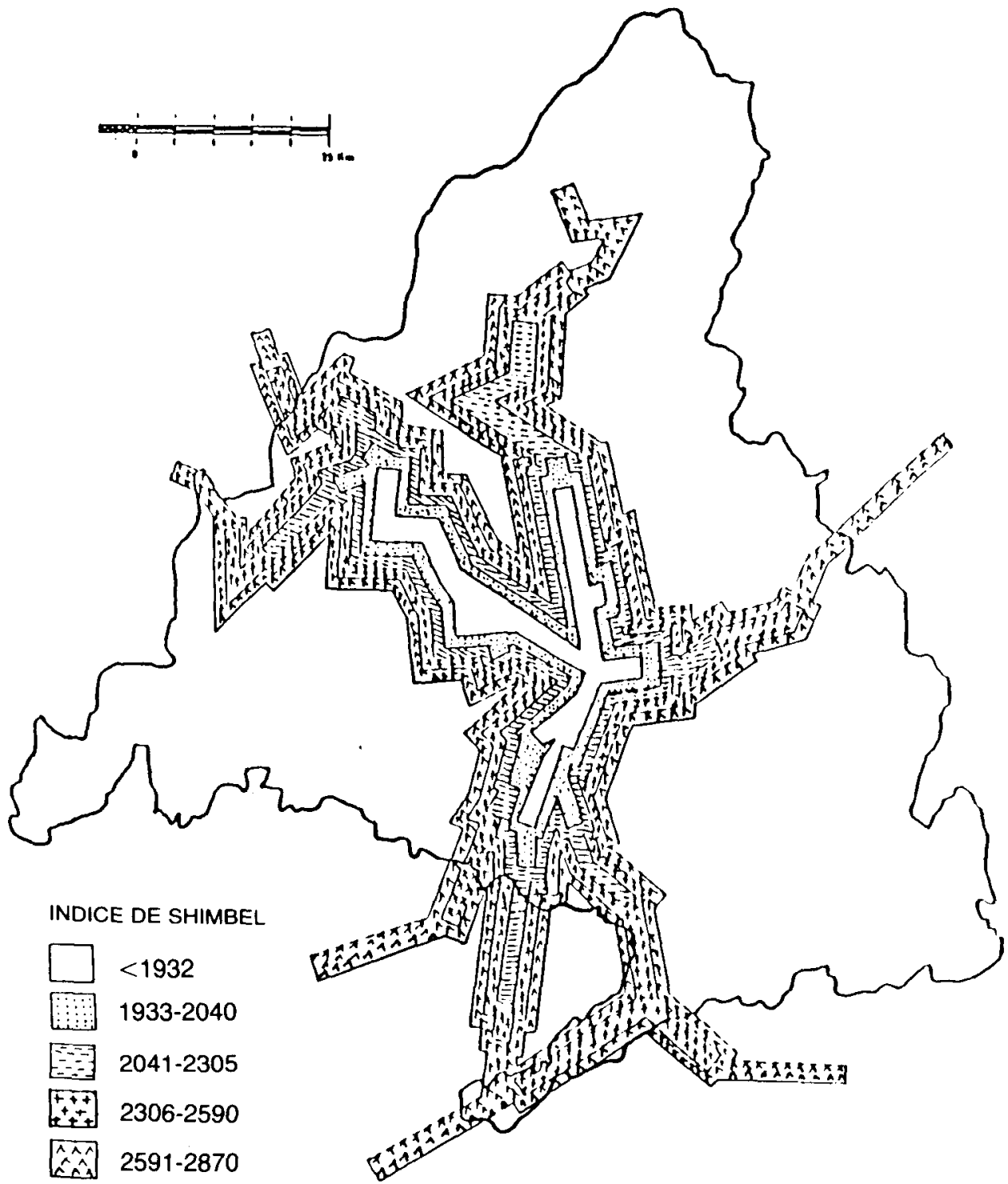

Fuente: Guias Comerciales de RENFE, 1984. (Elaboración propia). 


\section{FIGURA 3}

A

CAPACIDAD NETA DE DESPLAZAMIENTO POR MUNICIPIO EN SERVICIOS FERROVIARIOS-1975

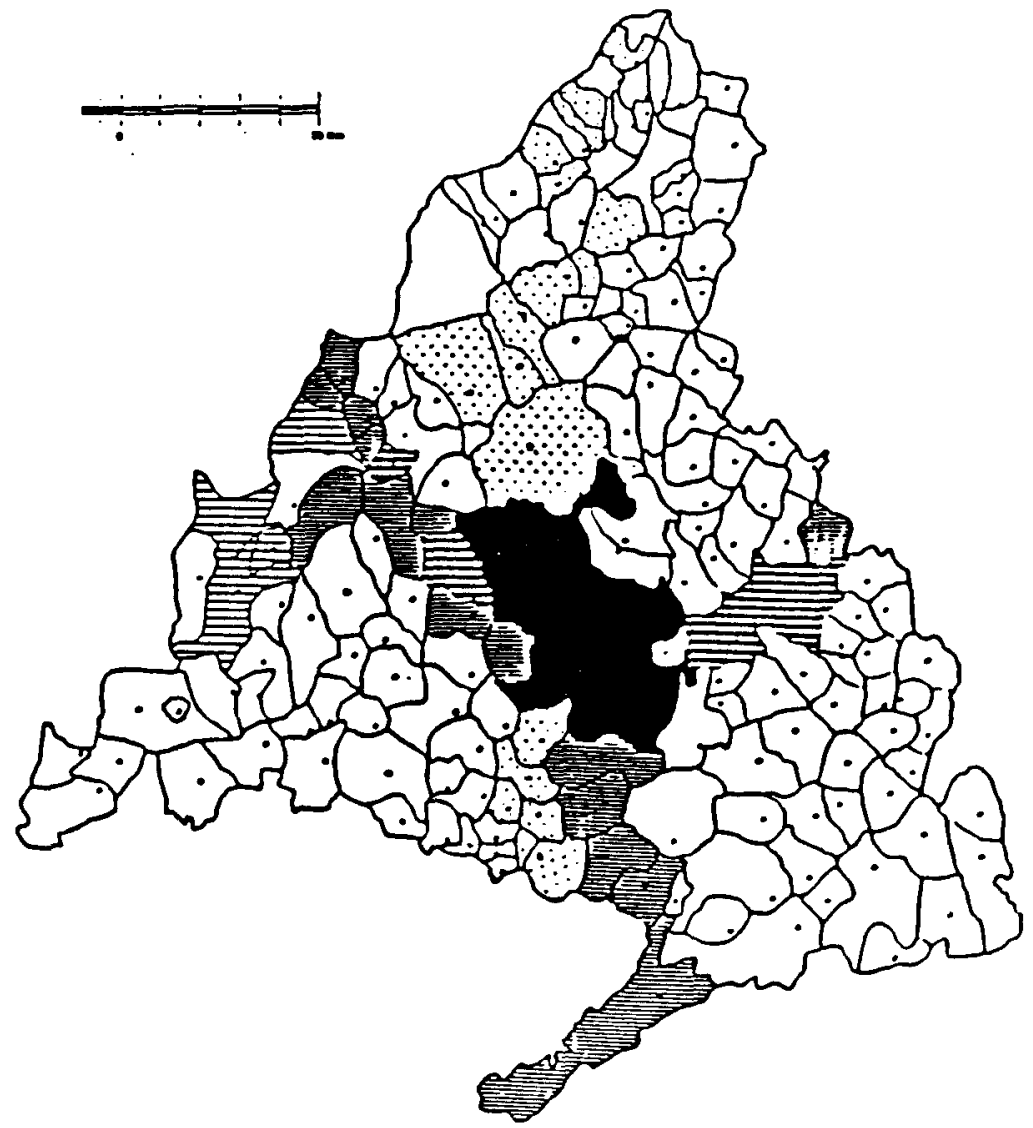

EXPEDICIONES COMPLETAS DISTINTAS

Diarias

$\square \quad 0-2$

$\nabla>2-<10$

㞎 $>10-<20$
Anuales

$0-730$

$>730-<3.650$

$>3.650-<7.300$
Diarias

武 $>20-<50$

$>50-<100$

$>100$
Anuales

$>7.300-<18.250$

$>18.250-<36.500$

$>36.500$

Fuente: Guias Comerciales de RENFE. (Elaboración propia). 
Una aproximación al análisis del balance entre oferta y demanda

FIGURA 3

$B$

CAPACIDAD NETA DE DESPLAZAMIENTO POR MUNICIPIO EN SERVICIOS FERROVIARIOS-1981

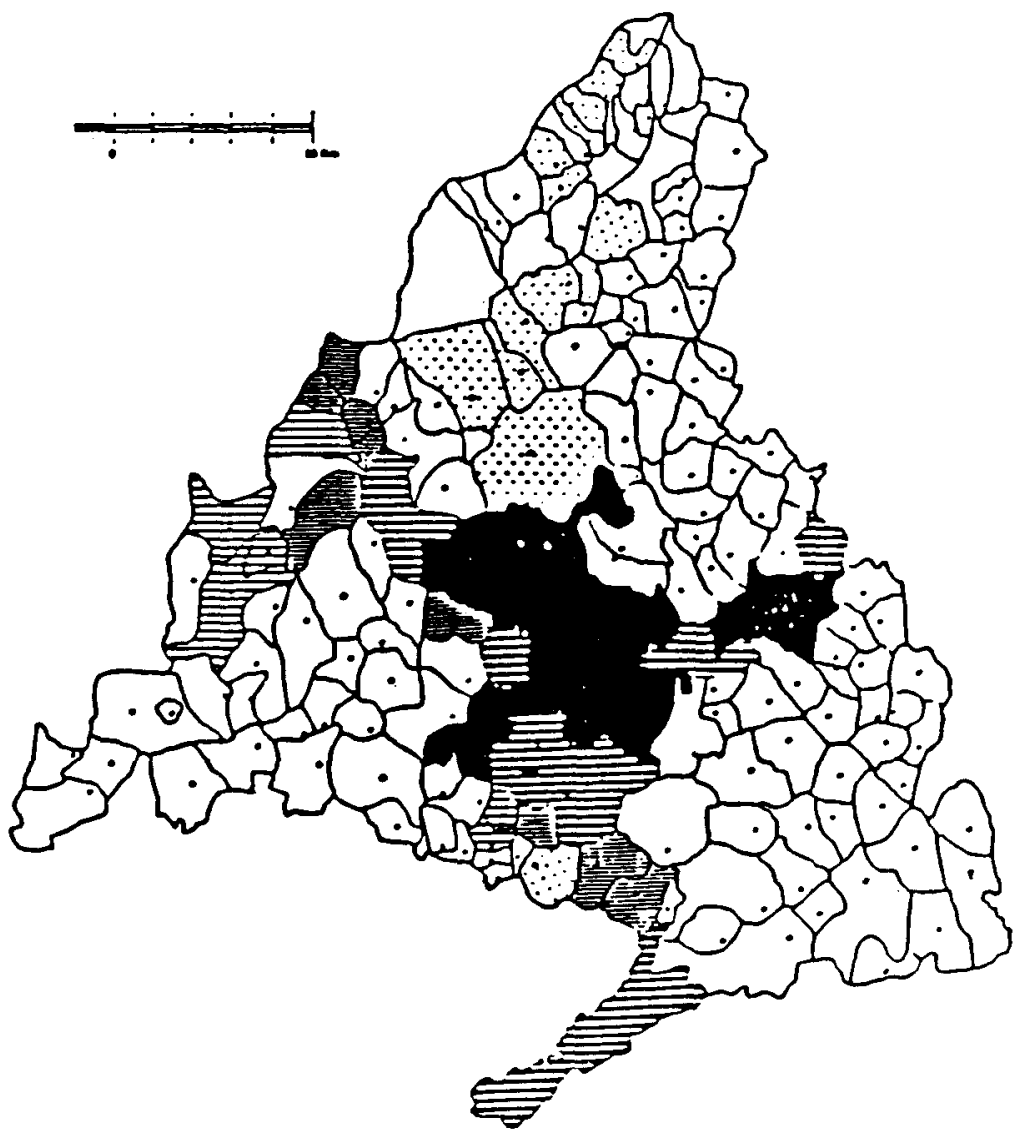

EXPEDICIONES COMPLETAS DISTINTAS

\begin{tabular}{lcccc}
\multicolumn{1}{r}{ Diarias } & Anuales & Diarias & Anuales \\
$\square$ & $0-2$ & $0-730$ & 娄 $>20-<50$ & $>7.300-<18.250$ \\
$\square>2-<10$ & $>730-<3.650$ & 目 $>50-<100$ & $>18.250-<36.500$ \\
$\square>10-<20$ & $>3.650-<7.300$ & $\square>100$ & $>36.500$
\end{tabular}

Fuente: Guias Comerciales de RENFE. (Elaboración propia). 
FIGURA 4

DISTRIBUCION ESPACIAL DE LOS RESIDUOS OBTENIDOS DEL ANALISIS DE REGRESION MULTIPLE PARA LA VARIABLE DEPENDIENTE OFERTA FERROVIARIA-1981

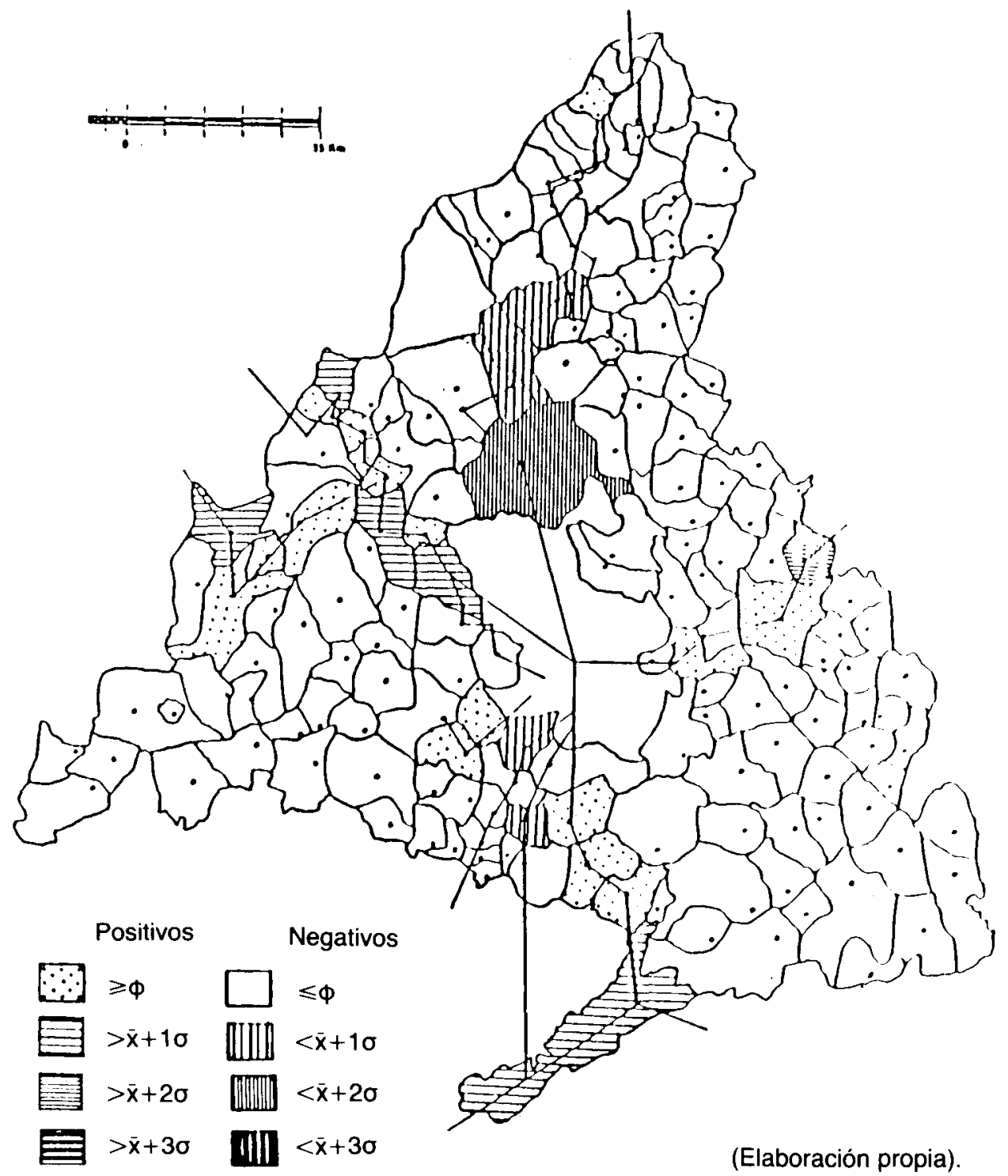


FIGURA 5

EVOLUCION DE LA TARIFA BASE DE RENFE

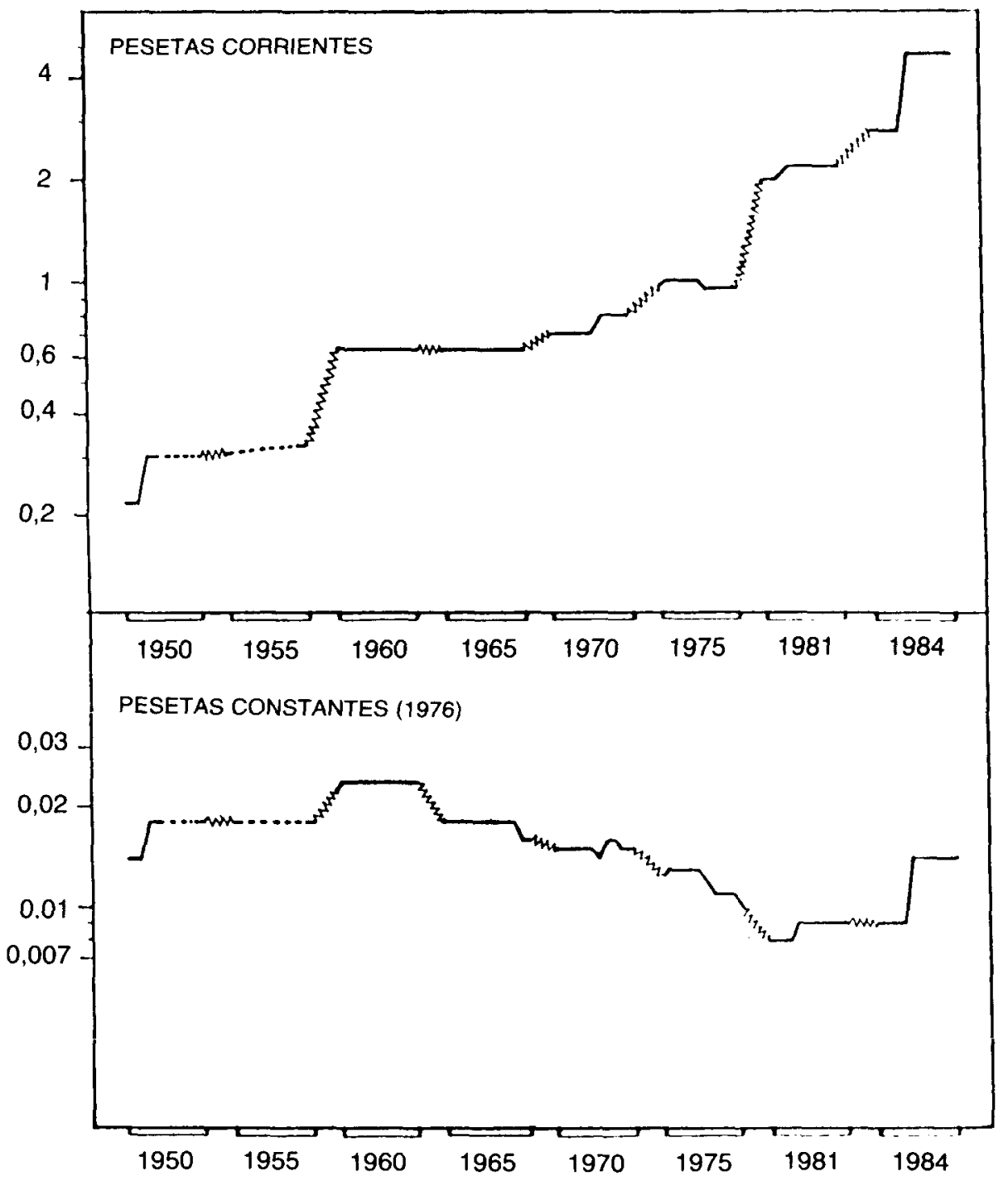

Representación semilogarítmica:

- ordenadas: tarifa base.

- abcisas: años.

Fuente: Guías Comerciales de RENFE. (Elaboración propia). 